\title{
Emil Theodor Kocher (1841-1917): contribuições para a Medicina e Neurocirurgia
}

Emil Theodor Kocher (1841-1917): contributions to Medicine and Neurosurgery

\author{
Henry Martins Soares Fortes ${ }^{1}$ (D), Marcelo Moares Valença ${ }^{2,3}$ (D) \\ ${ }^{1}$ Universidade de Pernambuco, Recife, Pernambuco, Brasil \\ ${ }^{2}$ Universidade Federal de Pernambuco, Recife, Pernambuco, Brasil \\ ${ }^{3}$ Unimed Recife, Recife, Pernambuco, Brasil
}

\section{$凶$}

Henry Martins Soares Fortes henrymsfortes@gmail.com

Edited by:

Juliana Ramos de Andrade

Palavras-chave:

Prêmio Nobel

Glândula Tireóide

Epilepsia

Cirurgiões

Suiça

Keywords:

Nobel Prize

Thyroid Gland

Epilepsy

Surgeons

Switzerland

\section{Resumo}

Theodor Kocher foi um grande cirurgião suíço que atuou no final do século XIX e início do século XX, contribuindo em diversas áreas da Medicina e sendo o primeiro cirurgião congratulado com um Prêmio Nobel de Medicina e Fisiologia, em 1909. Durante sua presidência da Clínica Cirúrgica da Universidade de Bern, Kocher se tornou referência entre os cirurgiões da Europa e influenciou grandes nomes da cirurgia, tais como Harvey Cushing e William Halsted. Além disso, seu rigor metodológico colaborou com a inserção do método científico na prática médica. Esse artigo de revisão propõe revisitar suas principais contribuições e discutir como seu trabalho influenciou no desenvolvimento da Medicina atual, com destaque para a cirurgia e fisiopatologia da tireóide, cirurgia da hipófise e cirurgia valvar para epilepsia.
Abstract
Theodor Kocher was a great Swiss surgeon who worked in the late nineteenth and early twentieth centuries, contributing to several areas of Medicine and being the first surgeon to be awarded a Nobel Prize in Medicine and Physiology in 1909. During his presidency at the University of Bern's Surgical Clinic, Kocher became a reference among surgeons in Europe and influenced great names in surgery, such as Harvey Cushing and William Halsted. Moreover, his methodological rigor collaborated with the insertion of the scientific method in medical practice. This review article proposes to revisit his main contributions and discuss how his work influenced the development of current medicine, with emphasis on surgery and pathophysiology of the thyroid, pituitary surgery, and valve surgery for epilepsy. 


\section{Introdução}

Emil Theodor Kocher nasceu no ano de 1841, em

EBern, na Suíça. ${ }^{1.3}$ Filho de Jacob Alexander Kocher, um engenheiro civil de sucesso, e de MariaWermuth Von Signau, pietista com forte convicção religiosa, Kocher teve o trabalho e a religiosidade como pontos fortes de sua criação. ${ }^{1}$ Com isso, ele se dedicou bastante à suas atividades acadêmicas como graduando na Universidade de Bern, obtendo o título de "Doctor of Medicine" em 1866.3.5

Fluente em inglês, alemão e francês, Kocher visitou grandes centros cirúrgicos e acadêmicos pela Europa, acompanhando Spencer Wells (1818-1897), em Londres, Rudolf Virchow (1821-1902), em Berlim, e passou um curto tempo na Universidade de Zurich, onde foi aluno de Theodor Billroth (1829-1894) e Bernhard von Langenbeck (1810-1887). ${ }^{4}$ Em suas passagens fora de Bern, Kocher foi introduzido à novas técnicas antissépticas, que ainda estavam em seus primórdios, e teve contato com uma importante literatura científica e com grandes nomes da Medicina da época.

Em seu retorno a Bern, Kocher obteve seu venia docendi, o licenciando para docência, e se tornou assistente do Prof. George Albert Lücke (1829-1884), professor chefe da Clínica Cirúrgica Universitária de Bern. ${ }^{3}$ Em 1872, Kocher sucedeu Lücke em seu cargo, permanecendo nele até sua morte. ${ }^{4} \mathrm{Em}$ sua gestão, a Clínica Cirúrgica de Bern passou de um hospital modesto para uma instituição de referência na Europa, atraindo grandes nomes da cirurgia, como William Halsted, Harvey Cushing e Charles Mayo. ${ }^{4}$

Kocher se diferenciava dos cirurgiões contemporâneos pela introdução de elementos novos na cirurgia, como a avaliação estatística de letalidade, complicações e resultados, a antissepsia e a técnica cirúrgica minuciosa, marcada por dissecções e hemostasias cuidadosas. ${ }^{3}$ Enquanto a grande maioria de seus colegas buscava a velocidade como sinal de sutileza na operação, ele buscava a precisão.

\section{Contribuições para a Fisiologia e Cirurgia da Tireóide}

No final do século XIX, a fisiologia da tireoide ainda era desconhecida e a cirurgia para retirada da glândula era temida e associada a alta taxa de mortalidade. Nesse cenário, a contribuição de sete cirurgiões apelidados de "Os Sete Magníficos da História da Tireoidectomia" mudou os paradigmas acerca desse procedimento. Dentre eles, Kocher teve o trabalho mais importante, sendo agraciado com ○ Prêmio Nobel de Medicina e Fisiologia, em 1909.5,7 Nesse sentido, ele foi o primeiro cirurgião congratulado com um prêmio Nobel. ${ }^{8,9}$

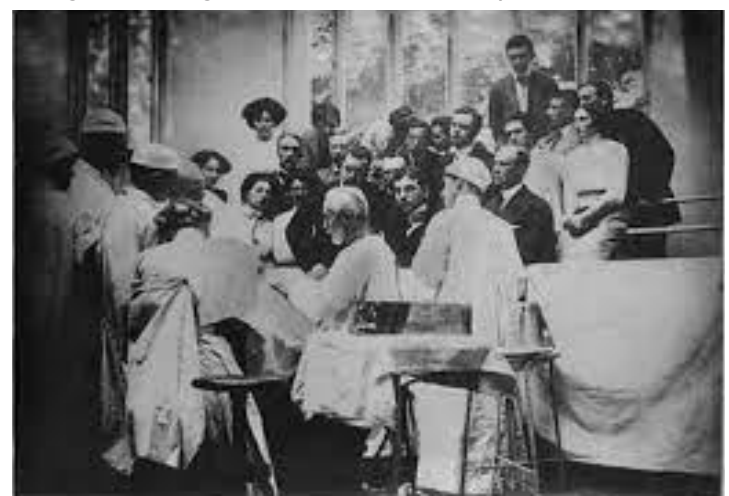

Figura 1. Kocher operando um aneurisma de aorta abdominal em 1911, William Halsted juntamente com seus discípulos auxiliando-o. ${ }^{3}$

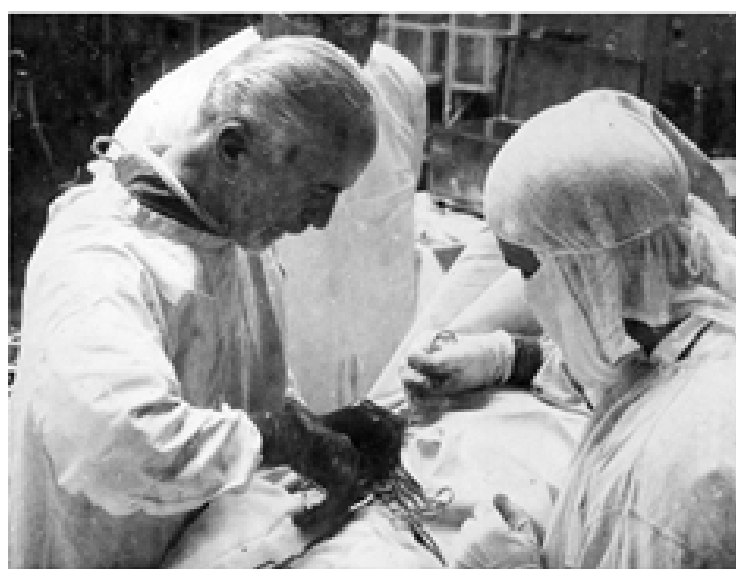

Figura 2. Theodor Kocher usando luvas de borracha em julho de 1914.6

Poucos anos após assumir a chefia da Clínica Cirúrgica, Kocher realizou uma série de 34 tireoidectomias, sendo que 16 dos pacientes apresentaram no pósoperatório um quadro de hipotireoidismo profundo, denominado por ele de "cachexia strumi priva". Após essa experiência, ele publicou o trabalho científico "Über die Ausrottung des Kropfes und seine Folgen" (Sobre a extirpação do bócio e suas consequências), onde foram discutidas as técnicas da tireoidectomia total e da lobectomia tireoidiana, que permanecem praticamente inalteradas até os dias de hoje. $8,10,11$

Baseado nisso, Kocher acreditava que a tireóide atuava na regulação da circulação sanguínea 
cerebral. Porém, com os achados de George Murray (1865-1939), em 1891, sobre aplicação de extrato de tireóide de ovelhas, e com seus próprios achados em 1892 sobre a ingestão de tecido tireoideano curar ou atenuar esse quadro de hipotireoidismo, essa sua primeira tese foi abandonada. ${ }^{8}$

Ainda no início dos anos 1900s, uma série de médicos (Hunziker, Bayard, Eggenberger) demonstraram que administração de iodo poderia prevenir o desenvolvimento de bócio. Em 1913, David Marine (1880-1976) passou um tempo em Bern discutindo casos com Kocher e ambos defenderam a tese de que a deficiência de iodo não era consequência do bócio, mas estava envolvida na sua fisiopatologia. ${ }^{3,8}$

Em 1909, Kocher foi agraciado com o Prêmio Nobel de Medicina e Fisiologia pelas suas contribuições na cirurgia, fisiologia e patologia da tireóide. $2,3,8,11,12$ Seu trabalho "Beunruhigende pathologische Manifestationen bei niedriggradiger Schilddrüsenerkrankung" (Manifestações patológicas preocupantes em doenças da tireóide de baixo grau) compreende esforços dele e de colegas no desenvolvimento da cirurgia e na investigação da fisiologia tireoidiana. Até o final de sua vida, Kocher teria realizado mais de 5 mil tireoidectomias, tendo letalidade de menos de $1 \%$, um grande avanço médico ao se considerar o cenário da época.

\section{Principais Contribuições Científicas}

Kocher realizou mais de 140 publicações, dentre as quais as principais estão na Tabela 1.

Tabela 1. Principais Publicações de Kocher

\begin{tabular}{|c|c|}
\hline Ano & Publicação \\
\hline 1870 & Eine neue Reduktionsmethode für Schulterverrenkung (Um novo método de redução para luxações do ombro) \\
\hline 1874 & Zur Pathologie und Therapie des Kopfes (Sobre a patologia e terapia do bócio) \\
\hline 1879 & Über Nervendehnung bei Trigeminusneuralgie (Sobre a tração nervosa na neuralgia do trigêmeo) \\
\hline 1881 & Die antiseptische Wundbehandlung (Tratamento antisséptico de feridas) \\
\hline 1887 & Die Krankheiten der männlichen Geschlechtsorgane (As doenças dos órgãos genitais masculinos) \\
\hline 1892 & Chirurgische Operations Lehre (Livro-texto cirúrgico) \\
\hline 1892 & $\begin{array}{l}\text { Zur Verhütung des Cretinismus und cretinoider Zustände nach neueren Forschungen (Para a prevenção de cretinismo e condições cretinóides, de acordo com pesquisas } \\
\text { recentes) }\end{array}$ \\
\hline 1892 & Chirurgische Beiträge zur Physiologie des Gehirns und Rückenmarks (Considerações cirúrgicas na fisiologia do cérebro e da medula espinal) \\
\hline 1895 & Vorlesungen über chirurgische Infektionskrankheiten (Palestras sobre doenças infecciosas cirúrgicas) \\
\hline 1896 & $\begin{array}{l}\text { Die Verletzungen der Wirbelsäule zugleich als Beitrag Zur Physiologie des menschlichen Rückenmarks(Trauma espinhal como contribuição para a fisiologia da medula } \\
\text { espinal) and Die Läsionen des Rückenmarks bei Verletzungen der Wirbelsäule (Lesões da medula espinal em trauma espinhal) }\end{array}$ \\
\hline 1896 & Beiträge zur Kenntniss einiger praktisch wichtiger Frakturformen (Contribuição para o conhecimento de algumas formas de fratura praticamente importantes) \\
\hline 1899 & Über einige Bedingungen operativer Heilung Der Epilepsie (Sobre alguns requisitos de cura cirúrgica na epilepsia) \\
\hline 1899 & Untersuchungen über die Bedingungen und Methodik operativer Druckentlastung des Gehirns (Estudos dos pré-requisitos e métodos de descompressão cirúrgica do cérebro) \\
\hline 1899 & Experimentelle Beiträge zur Ätiologie und Therapie Der Epilepsie (Contribuições experimentais para a etiologia e terapia da epilepsia) \\
\hline 1901 & Gehirnerschütterung, Hirndruck und chirurgische Eingriffe bei Hirnkrankheiten (Concussão cerebral, pressão intracraniana e intervenções cirúrgicas em doenças do cérebro) \\
\hline 1909 & Ein Fall von Hypophysis-Tumor mit operative Heilung (Cura cirúrgica em caso de tumor hipofisário) \\
\hline 1916 & $\begin{array}{l}\text { Zwei Fälle glücklich operierter grosser Hirntumoren Nebst Beiträgen zur Beurteilung organisch bedingter Epilepsie (Dois casos de tumores cerebrais gigantes operados } \\
\text { com sucesso e contribuições para a avaliação da epilepsia física) }\end{array}$ \\
\hline
\end{tabular}

\section{Estudo sobre etiologia e tratamento de epilepsia}

No final do século XIX, estava em alta o debate sobre a localização das funções cerebrais. John Hughlings Jackson (1835-1911) teorizou a epilepsia focal como uma manifestação de irritação cortical. Isso fez muitos pensarem em uma cirurgia sobre o córtex cerebral como tratamento. Em 1881, Kocher realizou sua primeira neurocirurgia visando o tratamento da epilepsia, o paciente teria sofrido uma fratura na região frontoparietal esquerda em um acidente ocorrido anos antes da intervenção. ${ }^{13}$ Após um período inicial sem crises convulsivas, o paciente teve suas crises epilépticas recidivadas, falecendo em 1888 após várias horas de um estado de mal epiléptico. ${ }^{13} \mathrm{Em}$ 1892, ele realizou uma lesionectomia cortical em um paciente de 8 anos de idade com crises epilépticas

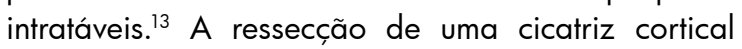
do lobo frontal direito sanou as crises da paciente.

Kocher percebeu que nos casos de traumatismo em que não se verificava defeitos visíveis externamente no crânio do paciente a localização da trepanação se tornava muito difícil. Diante disso, ainda em 1892 foi publicado seu aclamado livro-texto cirúrgico "Chirurgische Operations Lehre", traduzido em diversas línguas. Na obra, além de discorrer sobre cirurgias de várias porções da cabeça, Kocher descreve suas técnicas de trepanação e apresenta seu craniômetro, instrumento primordial para localização de estruturas ou lesões intracranianas. Kocher, nesse ponto, foi influenciado pelos achados e publicação de Sir Victor Alexander Haden Horsley (1857-1016), seu amigo de Londres, Inglaterra. ${ }^{3,13}$ 
Essa ferramenta consistia em dois feixes resilientes e uma fita elástica, posicionada na região frontal e occipital do crânio para obter uma fixação. Os pontos e linhas do craniômetro com relações e ângulos fixos entre si eram atribuídos de forma reprodutível a determinadas regiões do córtex, como córtex motor, giro supramarginal, giro angular, fissura parieto-occipital e fissura Silviana. Esse método foi desenvolvido com base no estudo anatômico de cérebros de cadáveres e das ilustrações de Victor Horsley (1857-1916). ${ }^{3}$

\section{Cirurgia da Hipófise}

As primeiras tentativas de cirurgia de hipófise por abordagem transesfenoidal foram dificultadas pela exposição limitada, iluminação deficiente e resultado estético insatisfatório. Porém, Kocher modificou a abordagem dessa cirurgia em 1909 ao optar pela ressecção do septo submucoso para expandir a visualização dentro da sela túrcica.10 Essa mudança foi muito importante, tanto pela redução do risco de infecção quanto pelo resultado estético mais atraente.

Harvey Cushing (1869-1939), por intermédio de William Halsted (1852-1922), passou 5 meses em Bern, onde teve contato direto com Kocher. ${ }^{14}$ Influenciado por ele e pelo trabalho de outros cirurgiões, Cushing executou a primeira incisão sublabial com ressecção submucosa do septo em 1910.10,14 Essa abordagem continuou a ser desenvolvida por seus alunos até se popularizar no final dos anos 50, com melhor iluminação e imagem intra-operatória.

\section{Outras Contribuições}

As contribuições de Kocher se estendem do desenvolvimento de ferramentas até o aperfeiçoamento

de abordagens cirúrgicas, tendo seu nome em vários instrumentos e manobras utilizados na prática médica (Tabela 2). Ele, juntamente com Joseph Lister (18271912) e Halsted, se destacaram como trio de cirurgiões responsáveis por introduzir uma técnica cirúrgica meticulosa, pautada em precisão, cuidado e técnicas antissépticas estritas, que impactou diretamente nos índices de letalidade de suas operações. ${ }^{14}$ Além disso, Kocher contribuiu para a formação de vários cirurgiões pela Universidade de Bern e também influenciou a Medicina com o uso de cientificismo ao medir os resultados de suas operações, buscando evidências para sua prática. ${ }^{14,15}$
Tabela 2. Epônimos associados a Kocher

\begin{tabular}{|c|c|}
\hline Ano & Publicação \\
\hline Epônimo & Descrição \\
\hline Pinça de Kocher & Pinça arterial com ganchos na extremidade. \\
\hline $\begin{array}{l}\text { Manobra de } \\
\text { Kocher }\end{array}$ & $\begin{array}{l}\text { Mobilização cirúrgica do duodeno para expor as porções } \\
\text { retroduodenal, intrapancreática e intraduodenal do ducto } \\
\text { biliar comum. }\end{array}$ \\
\hline Incisão de Kocher & $\begin{array}{l}\text { Incisão subcostal realizada no lado direito, expondo a } \\
\text { vesícula biliar e o ducto biliar comum, e no lado esquerdo } \\
\text { dando acesso à esplenectomia ou anastomose venosa } \\
\text { esplenorrenal. }\end{array}$ \\
\hline Incisão de Kocher II & Incisão transversa sobre a tireoide para remoção glandular. \\
\hline $\begin{array}{l}\text { Incisão de arco e } \\
\text { Kocher }\end{array}$ & Incisão oblíqua para abrir a articulação do joelho. \\
\hline Sinal de Kocher & $\begin{array}{l}\text { Verificação de assincronismo oculopalpebral em } \\
\text { portadores de hipotireoidismo. }\end{array}$ \\
\hline Ponto de Kocher & $\begin{array}{l}\text { Ponto localizado } 3 \mathrm{~cm} \text { lateral à linha média e } 1 \mathrm{~cm} \text { anterior } \\
\text { à sutura coronária, na linha hemipupilar ipsilateral. }\end{array}$ \\
\hline Reflexo de Kocher & $\begin{array}{l}\text { Contração dos músculos abdominais subsequente à } \\
\text { compressão exploratória do testículo. }\end{array}$ \\
\hline Método de Kocher & Método de fixação do útero à parede abdominal. \\
\hline $\begin{array}{l}\text { Método de Kocher } \\
\text { II }\end{array}$ & $\begin{array}{l}\text { Método para reduzir luxações do ombro (método de } \\
\text { rołação). }\end{array}$ \\
\hline $\begin{array}{l}\text { Síndrome de } \\
\text { Kocher-Debré- } \\
\text { Semelaigne }\end{array}$ & $\begin{array}{l}\text { Síndrome de hipotireoidismo associada ao aumento } \\
\text { muscular para dar a aparência de um Hércules infantil. }\end{array}$ \\
\hline
\end{tabular}

\section{Conclusão}

Concluímos este artigo informando que não temos como objetivo discorrer sobre toda vida e obra de Kocher, dada sua extensa produção que não pode ser contida em um único artigo, mas destacar suas principais contribuições para a evolução da Medicina. Desde avanços na neurocirurgia, cirurgia abdominal e cirurgia da tireóide à introdução de avaliação estatística e técnicas antissépticas, sua influência nas mais diversas áreas médicas é imensa e imprescindível para o avanço da Medicina e Ciência de sua época.

Henry Martins Soares Fortes

https://orcid.org/ 0000-0003-3963-9058

Marcelo Moares Valença

https://orcid.org/0000-0003-0678-3782

\section{Referências}

1. Dubhashi SP, Subnis BM and Sindwani RD. Theodor e. Kocher. Indian J Surg 2013;75(5):383-384 Doi:10.1007/s12262-012-0469-9

2. Bumbasirević MZ, Zagorac SG and Lesić AR. Emil Theodor Kocher (1841-1917)--orthopaedic surgeon and the first surgeon Nobel Prize winner. Acta Chir lugosl 2013;60(3):7-11 Doi:10.2298/ aci $1303007 b$ 
3. Hildebrandt G, Surbeck W and Stienen MN. Emil Theodor Kocher: the first Swiss neurosurgeon. Acta Neurochir (Wien) 2012;154(6):1105-1115; discussion 1115 Doi:10.1007/s00701-012-1341-1

4. Chigot JP. Theodor Emil Kocher, modern surgery pioneer. Ann Chir 2000; 125(9):884-892

5. Wiese ER and Gilbert JE. Theodore Kocher. Ann Med Hist 1931;3(5):521-529

6. Schlich T. Negotiating technologies in surgery: the controversy about surgical gloves in the 1890s. Bull Hist Med 2013;87(2):170-197 Doi:10.1353/ bhm.2013.0026

7. Kopp P. Theodor kocher (1841-1917) Nobel prize centenary 2009. Arq Bras Endocrinol Metabol 2009;53(9): $1176-1180 \quad$ Doi:10.1590/s000427302009000900015

8. Ellis $H$. Theodor Kocher: the first surgeon to be awarded the Nobel Prize. $\mathrm{Br} J$ Hosp Med (Lond) 2009;70(3):157 Doi:10.12968/ hmed.2009.70.3.40569

9. Kennie R. The Nobel surgeon: Theodor Kocher (1841-1917). J Invest Surg 1999;12(4):177-178 Doi: $10.1080 / 089419399272449$
10. Lanzino $G$ and Laws ER, Jr. Pioneers in the development of transsphenoidal surgery: Theodor Kocher, Oskar Hirsch, and Norman Dott. J Neurosurg 2001;95(6):1097-1103 Doi:10.3171/ jns.2001.95.6.1097

11. Ven Fong Z, Rosato EL, Lavu H, Yeo CJ and Cowan SW. Emil Theodor Kocher, M.D., and His Nobel Prize (1841-1917). 2012;78(12):1322-1324 Doi: $10.1177 / 000313481207801222$

12. Gulec S. The Art and Science of Thyroid Surgery in the Age of Genomics: 100 years after Theodor Kocher. Mol Imaging Radionud Ther 2017;26(Suppl 1):1-9 Doi: 10.4274/2017.26.suppl.01

13. Surbeck W, Stienen $M N$ and Hildebrandt G. Emil Theodor Kocher--valve surgery for epilepsy. Epilepsia 2012;53(12):2099-2103Ｄoi:10.1111/i.1528$1167.2012 .03663 . x$

14. Modlin IM. Surgical triumvirate of Theodor Kocher, Harvey Cushing, and William Halsted. World J Surg 1998;22(1):103-113 Doi:10.1007/s002689900357

15. Rutkow IM. William Halsted and Theodor Kocher: "an exquisite friendship". Ann Surg 1978;188(5):630637 Doi:10.1097/00000658-19781 1000-00008 